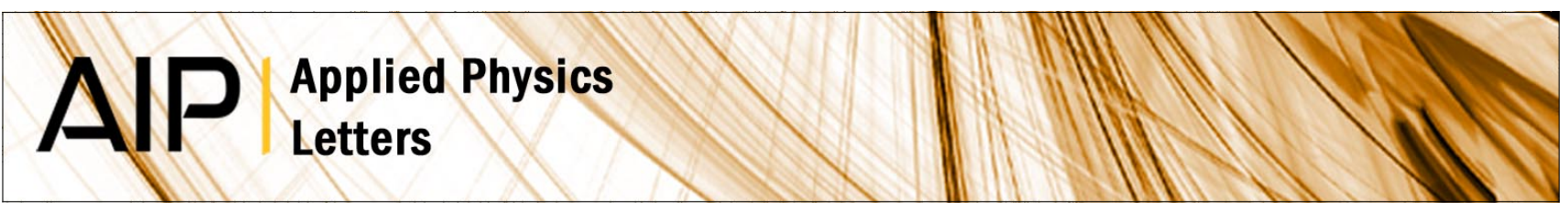

\title{
Low-field mobility in ultrathin silicon nanowire junctionless transistors
}

Bart Sorée, Wim Magnus, and William Vandenberghe

Citation: Appl. Phys. Lett. 99, 233509 (2011); doi: 10.1063/1.3669509

View online: http://dx.doi.org/10.1063/1.3669509

View Table of Contents: http://apl.aip.org/resource/1/APPLAB/v99/i23

Published by the American Institute of Physics.

\section{Related Articles}

2-bit operation based on modulated Fowler-Nordheim tunneling in charge-trapping flash memory cell Appl. Phys. Lett. 100, 052107 (2012)

Fabrication and characterization of ambipolar devices on an undoped $\mathrm{AIGaAs} / \mathrm{GaAs}$ heterostructure Appl. Phys. Lett. 100, 052101 (2012)

Improved photoresponse of InAs/GaAs quantum dot infrared photodetectors by using GaAs1-xSbx strain reducing layer

Appl. Phys. Lett. 100, 043512 (2012)

Local charge transfer doping in suspended graphene nanojunctions

Appl. Phys. Lett. 100, 023306 (2012)

Local charge transfer doping in suspended graphene nanojunctions

APL: Org. Electron. Photonics 5, 18 (2012)

\section{Additional information on Appl. Phys. Lett.}

Journal Homepage: http://apl.aip.org/

Journal Information: http://apl.aip.org/about/about_the_journal

Top downloads: http://apl.aip.org/features/most_downloaded

Information for Authors: http://apl.aip.org/authors

\section{ADVERTISEMENT}

\section{NEW!}

iPeerReview

AlP's Newest App

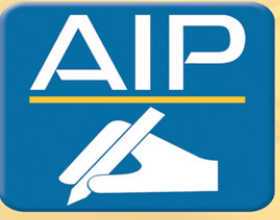

Authors...

Reviewers...

Check the status of

submitted papers remotely!

ADPublishing 


\title{
Low-field mobility in ultrathin silicon nanowire junctionless transistors
}

\author{
Bart Sorée, ${ }^{1,2, a)}$ Wim Magnus, ${ }^{1,2}$ and William Vandenberghe ${ }^{1,3}$ \\ ${ }^{1}$ IMEC, Kapeldreef 75, B-3001 Leuven, Belgium \\ ${ }^{2}$ Universiteit Antwerpen, Physics Department, Groenenborgerlaan 171, B-2020 Antwerpen, Belgium \\ ${ }^{3}$ Katholieke Universiteit Leuven, Department of Electrical Engineering, Kasteelpark Arenberg 10, \\ B-3001 Leuven, Belgium
}

(Received 8 August 2011; accepted 18 November 2011; published online 9 December 2011)

\begin{abstract}
We theoretically investigate the phonon, surface roughness and ionized impurity limited low-field mobility of ultrathin silicon n-type nanowire junctionless transistors in the long channel approximation with wire radii ranging from 2 to $5 \mathrm{~nm}$, as function of gate voltage. We show that surface roughness scattering is negligible as long as the wire radius is not too small and ionized impurity scattering is the dominant scattering mechanism. We also show that there exists an optimal radius where the ionized impurity limited mobility exhibits a maximum. (C) 2011 American Institute of Physics. [doi:10.1063/1.3669509]
\end{abstract}

A few years ago, the junctionless nanowire transistor (JNT) or pinch-off nanowire was proposed by several research groups ${ }^{1-4}$ and was recently fabricated. ${ }^{5}$ The JNT is a uniformly doped nanowire with no junctions, i.e. source, channel and drain are doped with the same doping type as schematically depicted in Fig. 1. The main motivation for introducing this device concept are the absence of doping junctions which makes the fabrication easier, ${ }^{4}$ and the reduction of detrimental interactions occurring at the interface between the silicon body of the wire and the insulator (surface roughness). ${ }^{1}$ Surface roughness scattering strongly depresses the mobility in a nanowire metal-oxide-semiconductor field-effect transistor (MOSFET) operating in strong inversion, since the inversion layer is prominently localized near the interface. The JNT however suffers less from surface roughness scattering because the majority carriers are distributed throughout the entire volume of the wire body. The working principle of the JNT requires sufficiently high doping levels in order to have an acceptable threshold voltage. As a result, ionized impurity scattering will be present which depresses the mobility considerably. ${ }^{1,4}$ It is commonly known $^{1-3,6,7}$ that for bulk JNTs with thicknesses larger than $10 \mathrm{~nm}$ the mobility agrees with the bulk mobility and is determined mainly by ionized impurity scattering for sufficiently high doping $\left(N_{\mathrm{D}}>10^{19} \mathrm{~cm}^{-3}\right)$. In the bulk case, the JNT is operating in bulk conduction mode and the ionized impurity scattering limited mobility is independent of gate voltage. In this paper, we do not rely on the bulk mobilities for the silicon JNT because we are considering ultrathin JNTs with a radius smaller than $5 \mathrm{~nm}$. As a result, we need to calculate the phonon, surface roughness and ionized impurity limited lowfield mobilities for ultrathin n-type silicon JNTs for the quantum confined majority charge carriers.

In order to calculate the low-field mobility for a $n$-type JNT we solve the self-consistent Poisson-Schrödinger problem for electrons in cylindrical coordinates $(r, z, \phi)$

$\overline{{ }^{a)} \text { Electronic mail: bart.soree@imec.be. }}$

$$
\begin{aligned}
& -\frac{\hbar^{2}}{2 m_{\alpha \perp}}\left[\frac{1}{r} \frac{d}{d r}\left(r \frac{d}{d r}\right)+\frac{1}{r^{2}} \frac{\partial^{2}}{\partial \phi^{2}}\right] \Psi(r, z, \phi) \\
& -\frac{\hbar^{2}}{2 m_{\alpha z}} \frac{\partial^{2} \Psi}{\partial z^{2}}+U(r, z, \phi) \Psi(r, z, \phi)=E \Psi(r, z, \phi),
\end{aligned}
$$

where $m_{\alpha \perp}=2 m_{\alpha x} m_{\alpha y} /\left(m_{\alpha x}+m_{\alpha y}\right), m_{\alpha x, y, z}$ are the effective masses of the twofold degenerate conduction band valleys $(\alpha=1,2,3)$ of silicon in the $x, y$ and $z$ directions. We have taken $m_{\mathrm{l}}=0.98 m_{\mathrm{e}}$ and $m_{\mathrm{t}}=0.19 m_{\mathrm{e}}$ for the longitudinal and transverse mass of the silicon conduction band. For a n-type JNT we can neglect the hole concentration and we only need to consider the conduction band electrons. Equation (1) contains a potential energy term $U(r, z, \phi)=-e \Phi(r, z, \phi)$ which is determined self consistently with Poisson's equation for the electrostatic potential

$$
\nabla^{2} \Phi=-\frac{\rho}{\epsilon_{\mathrm{Si}}}=-\frac{1}{\epsilon_{\mathrm{Si}}}\left[N_{\mathrm{D}}^{+}(r)-n(r)\right] .
$$

In our calculation we make the long-channel approximation, and we assume axial symmetry. As a result, the potential energy term as well as the charge density are only dependent on the radial coordinate $r$. The wave function is factorized as $\Psi_{k n m \alpha}(r, \phi, z)=F_{n m \alpha}(r) e^{i m \phi} e^{i k z}(m=0, \pm 1, \pm 2, \ldots)$ and the

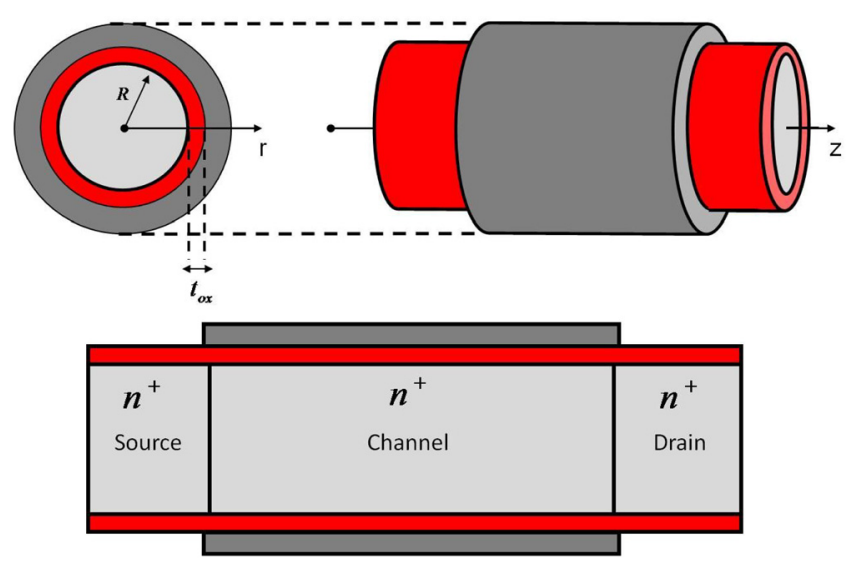

FIG. 1. (Color online) Schematical representation of the junctionless nanowire transistor 
self-consistent Poisson-Schrödinger problem reduces to calculating the radial part $F(r)$ of the wave function. The electron density is obtained from

$$
n(r)=2 \times \sum_{k v \alpha} g_{\alpha}\left|F_{v \alpha}(r)\right|^{2} f_{\mathrm{e}}\left(E_{\mathrm{C}}+E_{v \alpha}(k)\right),
$$

where $f_{\mathrm{e}}(E)=1 /\left(1+\exp \left(\beta_{\mathrm{e}}\left(E-E_{\mathrm{F}}\right)\right)\right)$ is the Fermi-Dirac distribution, while $E_{\mathrm{C}}$ is the conduction band minimum, $g_{\alpha}$ is the valley degeneracy, while $v$ is shorthand for the quantum numbers $n, m$. The equilibrium Fermi level $E_{\mathrm{F}}$ is determined in flatband condition by requiring global charge neutrality in the wire. We use the following expression for the low-field mobility: ${ }^{8,9}$

$$
\begin{aligned}
\mu= & \frac{4 e}{2 k T n} \sum_{v \alpha} g_{\alpha} \int_{E_{v \alpha}}^{\infty} d E \rho_{1 \mathrm{D}}^{v \alpha}(E) \tau_{v \alpha}(E)\left(v_{z}^{v \alpha}(E)\right)^{2} \\
& \times f_{v \alpha}^{0}(E)\left[1-f_{v \alpha}^{0}(E)\right] .
\end{aligned}
$$

where $n=\frac{2}{L} \sum_{v \alpha k} g_{\alpha} f_{v \alpha}^{0}(k)$ is the linear electron density, $v_{z}^{v \alpha}(k)=d E_{v \alpha}(k) / d(\hbar k)$ is the group velocity, $\tau_{v \alpha}(k)$ is the relaxation time, and $f_{\nu \alpha}^{0}(k)$ is the equilibrium distribution function for the $v$-th subband and $\alpha$-th valley. The function $\rho_{1 \mathrm{D}}^{v \alpha}(E)$ is the one-dimensional density of states

$$
\rho_{1 \mathrm{D}}^{v \alpha}(E)=\frac{1}{\pi \hbar} \sqrt{\frac{m_{\alpha z}}{2\left(E-E_{v \alpha}\right)}}
$$

for $E>E_{v \alpha}$ and zero elsewhere. The relaxation rate $1 / \tau_{v \alpha}(E)$ is obtained from the Boltzmann equation in the relaxation time approximation

$$
\begin{aligned}
\frac{1}{\tau_{v \alpha}(k)}= & \sum_{k^{\prime} v^{\prime} \alpha^{\prime}} S\left(k^{\prime} v^{\prime} \alpha^{\prime}, k v \alpha\right) \frac{1-f_{v \alpha}^{0}\left(E^{\prime}\right)}{1-f_{v \alpha}^{0}(E)} \\
& \times\left(1-\frac{v_{z}^{v^{\prime} \alpha^{\prime}}\left(k^{\prime}\right) \tau_{v^{\prime} \alpha^{\prime}}\left(k^{\prime}\right)}{v_{z}^{v \alpha}(k) \tau_{v \alpha}(k)}\right) .
\end{aligned}
$$

The microscopic scattering rate $S\left(k^{\prime} v^{\prime} \alpha^{\prime}, k v \alpha\right)$ is obtained from Fermi's golden rule. ${ }^{10}$ We consider phonon, surface roughness and ionized impurity scattering as the main scattering agents. The phonon scattering includes both acoustic

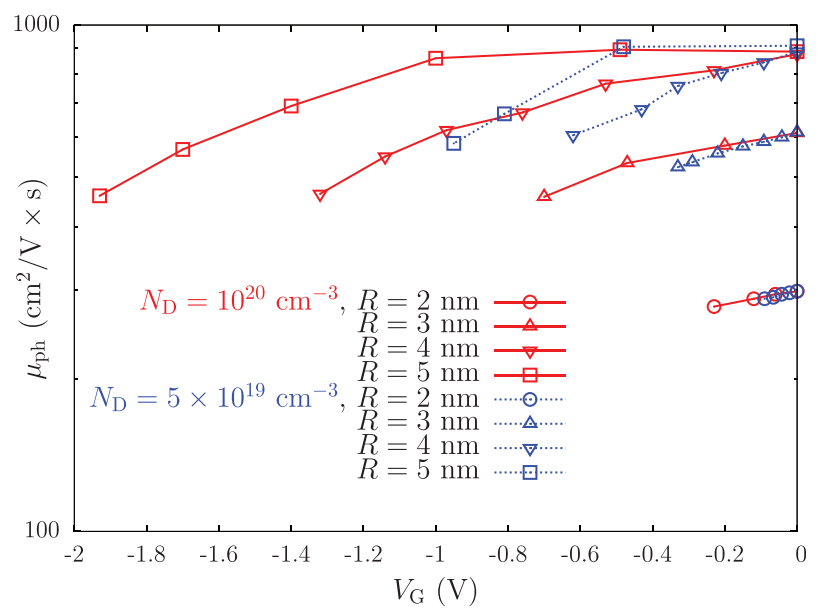

FIG. 2. (Color online) Phonon limited mobility as a function of gate voltage for $N_{\mathrm{D}}=10^{20} \mathrm{~cm}^{-3}$ (red) and $N_{\mathrm{D}}=5 \times 10^{19} \mathrm{~cm}^{-3}$ (blue) and wire radii $R=2,3,4$, and $5 \mathrm{~nm}$.

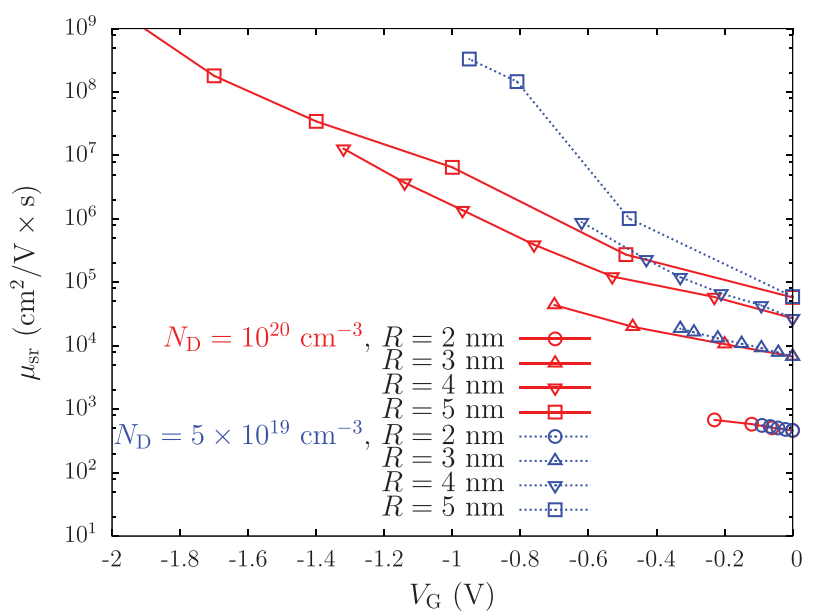

FIG. 3. (Color online) Surface roughness limited mobility as a function of gate voltage for $N_{\mathrm{D}}=10^{20} \mathrm{~cm}^{-3}$ (red) and $N_{\mathrm{D}}=5 \times 10^{19} \mathrm{~cm}^{-3}$ (blue) and wire $\operatorname{radii} R=2,3,4$, and $5 \mathrm{~nm}$.

and nonpolar optical phonon scattering where we have used the same values for the deformation potentials and sound velocity as in Refs. 10 and 11 . The scattering rate taking into account ionized impurities is calculated from the matrix element with the electrostatic potential accompanying a point charge surrounded by a dielectric and a metal gate. For the surface roughness scattering we considered an approach similar to Ref. 10 where we have used $\Lambda=1 \mathrm{~nm}$ for the correlation length and $\Delta=0.5 \mathrm{~nm}$ for the variance of the step height. In Figs. 2-4 we have plotted the phonon, ionized impurity and surface roughness limited mobility as a function of the applied gate voltage for a doping density of $N_{\mathrm{D}}=10^{20} \mathrm{~cm}^{-3}$ and $N_{\mathrm{D}}=5 \times 10^{19} \mathrm{~cm}^{-3}$, oxide thickness $t_{\mathrm{ox}}=1 \mathrm{~nm}$, and wire radii ranging from $R=2 \mathrm{~nm}$ to $R=5 \mathrm{~nm}$. The gate voltage sweep for each radius and doping density ranges from $V_{\mathrm{G}}=0$ (flatband) to $V_{\mathrm{G}}=V_{\mathrm{T}}$ (full depletion or pinch-off). The threshold or pinch-off voltage $V_{\mathrm{T}}$ is a function of wire radius, oxide thickness, and doping density. ${ }^{1,2}$

Fig. 2 shows that as the wire radius becomes smaller or the gate voltage is made more negative, the phonon limited mobility decreases due to an increase of the form factors. The same conclusion was reached for a cylindrical nanowire MOSFET. ${ }^{10}$ For $R>4 \mathrm{~nm}$ and $V_{\mathrm{G}}=0$ the phonon limited

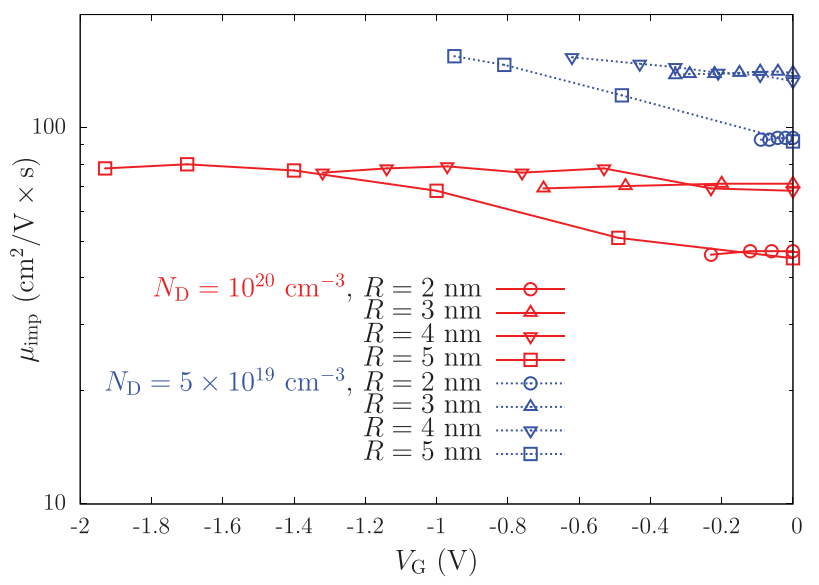

FIG. 4. (Color online) Ionized impurity limited mobility as a function of gate voltage for $N_{\mathrm{D}}=10^{20} \mathrm{~cm}^{-3}$ (red) and $N_{\mathrm{D}}=5 \times 10^{19} \mathrm{~cm}^{-3}$ (blue) and wire radii $R=2,3,4$ and $5 \mathrm{~nm}$. 


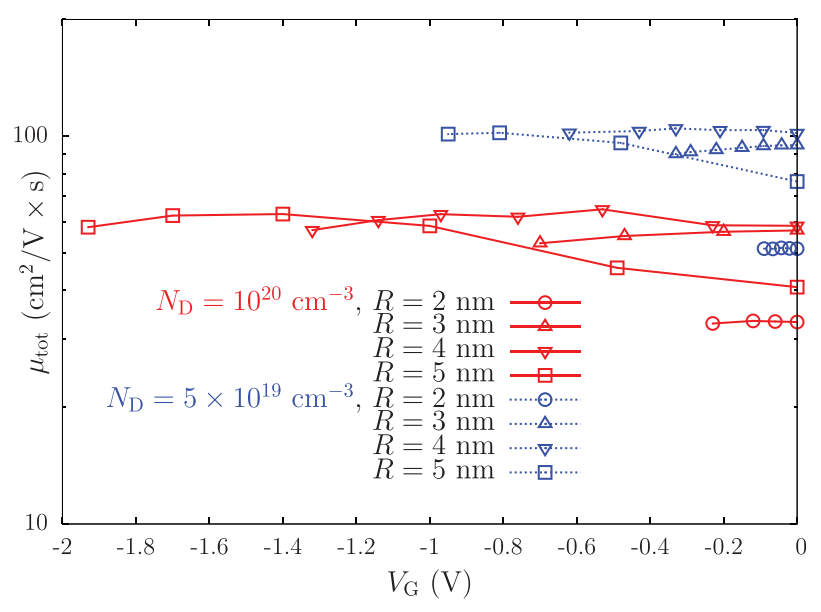

FIG. 5. (Color online) Total mobility as a function of gate voltage for $N_{\mathrm{D}}=10^{20} \mathrm{~cm}^{-3}$ (red) and $N_{\mathrm{D}}=5 \times 10^{19} \mathrm{~cm}^{-3}$ (blue) and wire radii $R=2,3,4$ and $5 \mathrm{~nm}$.

mobility converges to the bulk phonon limited mobility and is in good agreement with results obtained in other references. ${ }^{10,12}$ The surface roughness limited mobility $\mu_{\text {sr }}$ shown in Fig. 3 strongly depends on wire radius and applied gate voltage. As the gate voltage is decreased from flatband $\left(V_{\mathrm{G}}=0\right)$ to full depletion $\left(V_{\mathrm{G}}=V_{\mathrm{T}}\right) \mu_{\mathrm{sr}}$ gradually and monotonically increases because the carriers (electrons) are pushed to the middle of the wire, i.e., away from the surface separating the silicon channel from the insulator thus making surface roughness less relevant. For a fixed wire radius, the lowest surface roughness value is obtained when $V_{\mathrm{G}}=0$. As long as the wire radius is larger than the average step height, i.e., $R \gg \Delta$, the surface roughness limited mobility is negligible for $R=3,4$, and $5 \mathrm{~nm}$ and all values of $V_{\mathrm{G}}$ as shown in Fig. 3. For $R=2 \mathrm{~nm}, \mu_{\mathrm{sr}}$ cannot be neglected anymore and must be taken into account when calculating the total mobility. In Fig. 4 we have plotted the ionized impurity limited mobility. This scattering mechanism dominates over surface roughness scattering as long as the wire is not too small $(R>\Delta)$. We

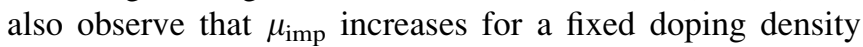
when the wire radius decreases from $R=5 \mathrm{~nm}$ to $R=4 \mathrm{~nm}$ reaching a maximum optimum value for a radius between 3 and $4 \mathrm{~nm}$ and decreases when going from $R=3 \mathrm{~nm}$ to $R=2 \mathrm{~nm}$. This effect can be ascribed to the behavior of the form factors associated with Coulomb scattering as the wire radius is decreased. The ionized impurity limited mobility shows a decreasing trend for $R \geq 4 \mathrm{~nm}$ as the gate voltage is increased, while for $R \leq 3 \mathrm{~nm}$ it remains almost constant. This behavior is due to the combined effect of confinement on screening, wave function overlap and density of states.
Finally, in Fig. 5 we have plotted the total mobility for doping densities $N_{\mathrm{D}}=5 \times 10^{19} \mathrm{~cm}^{-3}$ and $N_{\mathrm{D}}=10^{20} \mathrm{~cm}^{-3}$ with $R=2,3,4$, and $5 \mathrm{~nm}$ as a function of gate voltage. The total mobility is dominated by ionized impurity scattering, except for the case where the wire radius is too small to neglect surface roughness scattering, i.e., for $R=2 \mathrm{~nm}$ or smaller and gate voltages much larger than the threshold voltage, i.e., $V_{\mathrm{G}} \gg V_{\mathrm{T}}$.

In conclusion, we have shown that surface roughness scattering plays a minor or negligible role for a JNT as long as the wire radius is sufficiently large, i.e., $R \gg \Delta$. In this case ionized impurity scattering is the dominant scattering mechanism for a sufficient high doping density. Contrary to the case of heavily doped and thick JNTs operating in bulk conduction mode we do observe a dependence of $\mu_{\mathrm{imp}}$ on $V_{\mathrm{G}}$ and $R$ due to confinement. Furthermore we find an optimal value of $R=2-3 \mathrm{~nm}$ where $\mu_{\mathrm{imp}}$ is maximal. For ultrathin JNTs with a radius $R$ comparable or smaller than the variance of the step height $\Delta$, surface roughness will become the dominant scattering mechanism, while ionized impurity scattering will still significantly contribute. The total mobility for JNTs with radii ranging between $R=2 \mathrm{~nm}$ and $R=5 \mathrm{~nm}$ and with doping densities ranging between $N_{\mathrm{D}}$ $=5 \times 10^{19} \mathrm{~cm}^{-3}$ and $N_{\mathrm{D}}=10^{20} \mathrm{~cm}^{-3}$ ranges from approximately $40 \mathrm{~cm}^{2} / \mathrm{V} \cdot \mathrm{s}$ up to $100 \mathrm{~cm}^{2} / \mathrm{V} \cdot \mathrm{s}$.

This work is supported by the EU project SQWIRE (FP7-ICT-STREP nr. 257111). William Vandenberghe gratefully acknowledges the Ph.D. stipend from the Institute for the Promotion of Innovation through Science and Technology in Flanders (IWT-Vlaanderen).

${ }^{1}$ B. Sorée, W. Magnus, and G. Pourtois, J. Computat. Phys. 7, 380 (2008).

${ }^{2}$ B. Sorée, W. Magnus, M. Szepieniec, W. Vandenberghe, A. Verhulst, G. Pourtois, G. Groeseneken, S. D. Gendt, and M. Heyns, ECS Trans. 28, 15 (2010).

${ }^{3}$ B. Sorée and W. Magnus, ULIS 2009 Proc. 2009, 245.

${ }^{4}$ C.-W. Lee, A. Afzalian, N. D. Akhavan, R. Yan, I. Ferain, and J.-P. Colinge, Appl. Phys. Lett. 94, 053511 (2009).

${ }^{5}$ J. Colinge, C. Lee, A. Afzalian, N. Akhavan, R. Yan, I. Ferain, P. Ravazi, B. O'Neill, A. Blake, M. White, A. Kelleher, B. McCarthy, and R. Murphy, Nat. Nanotechnol. 5, 225 (2010).

${ }^{6}$ J. Raskin, J. Colinge, I. Ferrain, A. Kranti, C. Lee, N. Akhavan, R. Yan, P. Razavi, and R. Yu, Appl. Phys. Lett. 97, 042114 (2010).

${ }^{7}$ C.-W. Lee, A. Borne, I. Ferain, A. Afzalian, R. Yan, N. Akhavan, P. Razavi, and J.-P. Colinge, IEEE Trans. Electron Devices 57, 620 (2010).

${ }^{8}$ R. Kubo, J. Phys. Soc. Jpn. 12, 570 (1957).

${ }^{9}$ D. A. Greenwood, Proc. Phys. Soc. London 71, 585 (1958).

${ }^{10}$ J. Seonghoon, M. Fischetti, and T.-W. Tang, J. Appl. Phys. 102, 083715 (2007).

${ }^{11}$ C. Jacoboni and L. Reggiani, Rev. Mod. Phys. 55, 645 (1983).

${ }^{12}$ E. B. Ramayya, D. Vasileska, S. M. Goodnick, and I. Knezevic, J. Appl. Phys. 104, 063711 (2008). 